\title{
Theoretical Investigation of PAHs: Implications to Diffuse Interstellar Bands
}

\author{
A. Pathak ${ }^{1}$, M. Buragohain ${ }^{1}$, M. Hammonds ${ }^{2}$ and P. J. Sarre ${ }^{2}$ \\ ${ }^{1}$ Department of Physics, Tezpur University, Napaam, Tezpur 784 028, India \\ email: amit@tezu.ernet.in \\ ${ }^{2}$ School of Chemistry, The University of Nottingham, University Park, Nottingham, \\ NG7 2RD, UK
}

\begin{abstract}
About a thousand optical absorption features on the interstellar extinction curve popularly known as the Diffuse Interstellar Bands (DIBs) have been observed. The numbers are increasing every year, thanks to the improvement in telescope and spectroscopic technology. Ultra-high resolution spectroscopic observations and emission features corresponding to some of the DIBs suggest that, some if not all, of these features are due to large molecules. The strength of DIBs depend on the amount of reddening which is directly proportional to the amount of material present between the background star and the observer. Since, the strengths of the DIBs are not strongly correlated with each other, there must be several carriers. Time Dependent Density Functional Theory (TDDFT) calculations are useful in narrowing down molecular systems that may be further investigated in the laboratory.

The observations of the unidentified infrared (UIR) bands point towards the widespread presence of Polycyclic Aromatic Hydrocarbon (PAH) molecules. Though, not a single PAH has been discovered in interstellar space, these are the largest molecules suspected to be present. PAHs are stable towards energetic environment prevailing under interstellar conditions rendering these molecules to be good candidates as DIB carriers. We report TDDFT calculations to predict electronic transitions of neutral, protonated-deuteronated and PAHs with five member rings with various sites of protonation and deuteronation. Compared to their neutral forms, these charged isoelectronic forms of PAHs are predicted to have active transitions in the visible region, which means they are suitable candidates as carriers for some of the DIBs and laboratory studies are warranted for these systems.
\end{abstract}

Keywords. molecular processes, ISM: lines and bands, ISM: molecules.

\section{Introduction}

Diffuse Interstellar Bands (DIBs) are optical absorption features caused by the absorption of light by interstellar matter observed towards highly reddened sources. They are considered to be interstellar in origin due to their independent nature of Doppler shift associated with stellar lines (Merrill et al. 1938). Mary Lea Heger discovered the first DIB (Heger 1922) and till date hundreds of DIBs have been identified, but the assignment of molecules responsible for these features is still elusive (Herbig 1995; Sarre 2006). High resolution spectroscopic observations have confirmed the presence of substructures in some of the DIBs which is a strong evidence in favour of the molecular origin of DIBs (Sarre 2006). Observations do not show any strong correlation between the strength of individual DIBs, pointing to the possibility of several carriers.

The ubiquity of polycyclic aromatic hydrocarbon $(\mathrm{PAH})$ molecules in astrophysical environments proposed by the observation of Unidentified Infrared (UIR) emission bands (Tielens 2008) has led to the hypothesis that PAHs might be the carrier of some of the DIBs (Salama et al. 1996, 2011). Recently protonated form of PAHs $\left(\mathrm{HPAH}^{+}\right.$) formed 


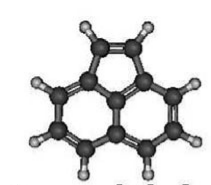

Acenaphthylene

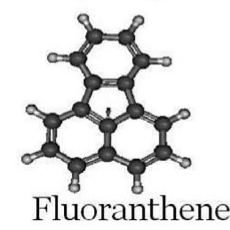

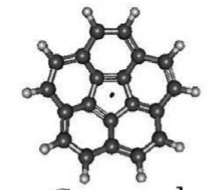

Corannulene

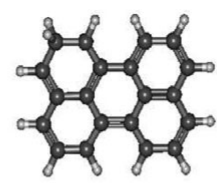

Perylene

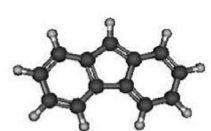

Fluorene
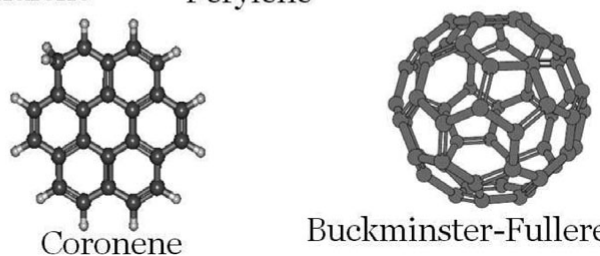

Buckminster-Fullerene $\mathrm{C}_{60}$

Figure 1. Sample PAHs, PAHs with five member rings and Buckminsterfullerene $\left(C_{60}\right)$.

by the addition of $\mathrm{H}$ to $\mathrm{PAH}$ cations has received attention as they show electronic transitions in the visible region where most DIBs are present (Pathak \& Sarre 2008; Hammonds et al. 2009).

The higher Lyman lines seen in the FUSE spectra towards several sources show the presence of deuterium in the interstellar medium (Hoopes et al. 2003). The detection of two infrared emission bands at 4.4 and $4.65 \mu \mathrm{m}$ in the interstellar spectra attributed to C-D stretching vibrations in PAHs carrying one or more D atoms point to the existence of deuterated-PAHs (dPAHs) (Peeters et al. 2004).

Protonated as well deuteronated PAHs might have a significant presence in the interstellar medium. Here we report electronic transitions in neutral PAHs and their protonated $\left(\mathrm{HPAH}^{+}\right)$and deuteronated $\left(\mathrm{dPAH}^{+}\right)$counterparts using Time Dependent Density Functional Theory (TDDFT). Our study also includes the PAHs with 5 member rings (Fig. 1).

\section{Electronic Spectra}

GAMESS quantum chemistry suite of programs (Schmidt et al. 1993) have been used to obtain the electronic transition of PAHs. The equilibrium geometries of molecules were obtained by optimizing the structures using DFT with a B3LYP/6-311G** basis. TDDFT with BLYP and a $6-311 \mathrm{G}^{* *}$ basis set is used to calculate the excitation energies and the oscillator strengths for electronic transitions.

Neither protonation nor deuteronation increases the number of electrons, but the protonated and deuteronated sites become $s p^{3}$ hybridized and the HOMO-LUMO gap is reduced. The electrons require less photo-excitation energy thereby showing transitions towards longer wavelength, sometimes in the visible region as compared to the neutrals.

Fig. 2(a) shows the calculated spectra for coronene $\left(\mathrm{C}_{24} \mathrm{H}_{12}\right)$ and its charged and isomeric counterparts. The neutral coronene spectrum shows a pair of degenerate transitions near $333 \mathrm{~nm}$ that have oscillator strengths of about 0.5. For protonated coronene, transitions appear in the near UV and the visible region, although weaker. The most striking difference is the appearance of strong features in the visible region near $618 \mathrm{~nm}$ and $731 \mathrm{~nm}$ with oscillator strengths of 0.07 and 0.04 respectively. Similar features in the visible are seen in the case of deuteronated PAHs.

PAHs with five member rings formed due to dehydrogenation and carbon loss of PAHs show similar transitions as regular PAHs (Fig. 2(b)). PAHs with five member rings are important as a predecessor to the formation of $C_{60}$. Therefore, the presence of $C_{60}$ in 


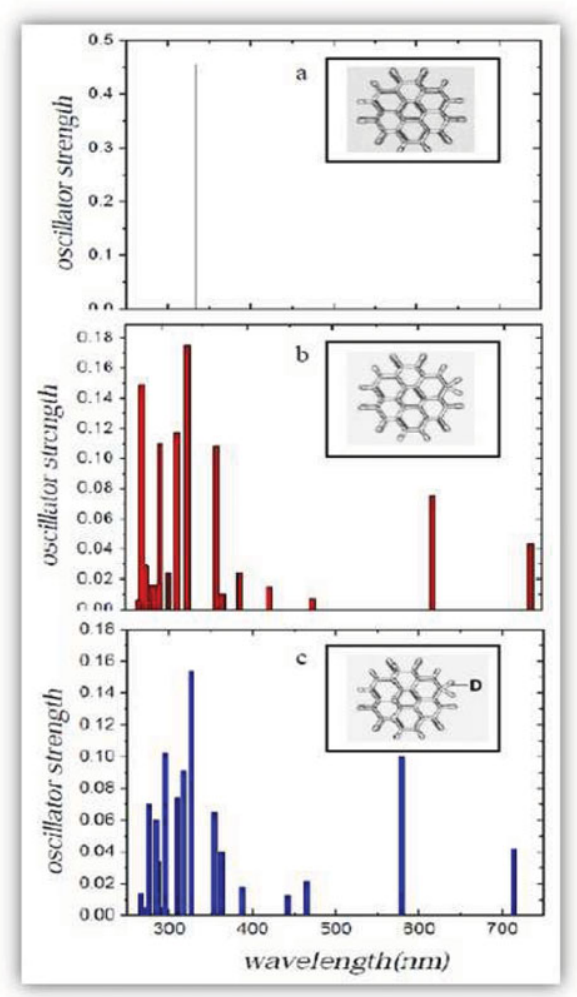

(a) Absorption spectra of neutral, protonated and deuteronated coronene (topbottom). D denotes the deuteronation site.

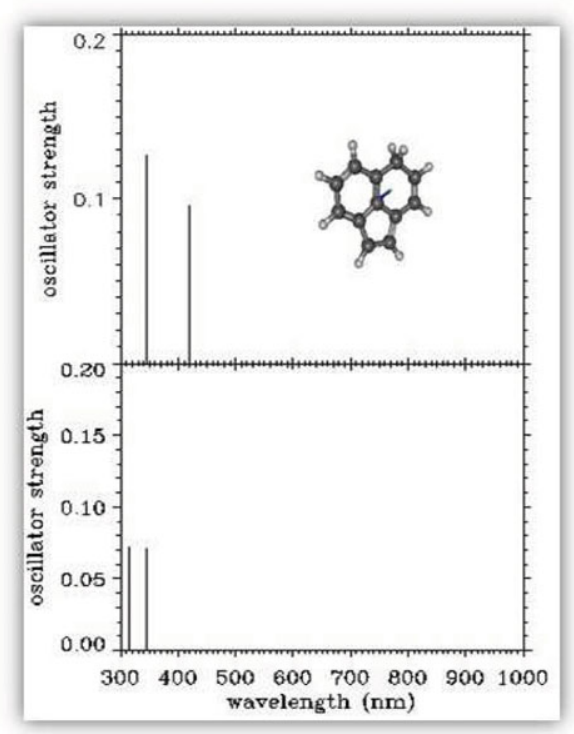

(b) Absorption spectra for protonated and neutral acenaphthylene (top-bottom).

Figure 2. Absorption spectra of coronene and acenaphthylene.

space (Cami et al. 2010) may be associated with the presence of PAHs with five-member rings.

\section{Summary}

It is observed that the protonated and deuteronated forms of PAHs show electronic transitions in the visible wavelength range compared to the corresponding PAH neutrals. With increasing molecular weight, such transitions become even significant. PAHs with five member rings are found to have similar transitions and may be possible carriers of DIBs. Based upon this result and other astrochemical properties, it is important that upcoming laboratory studies include protonated, deuteronated and PAHs with five member rings to enable a better understanding of the DIB carriers.

\section{References}

Cami, J., Bernard-Salas, J., Peeters, E., \& Malek, S. E. 2010, Science, 329, 1180

Hammonds, M., Pathak, A., \& Sarre, P. J. 2009, Phys. Chem. Chem. Phys., 11, 4458

Heger, M. L. 1922, Lick Observatory Bull, 337, 141

Herbig, G. H. 1995, ARA\&A, 33, 19

Hoopes, C. G., Sembach, K. R., Hbrard, G., Moos, H. W., \& Knuth, D. C. 2003, ApJ, 586, 1094

Merrill, P. W. \& Wilson, O. C. 1938, ApJ, 87, 9 
Pathak, A. \& Sarre, P. J. 2008, MNRAS, 391, L10

Peeters, E., Allamandola, L. J., Bauschlicher, Jr. C. W., Hudgins, D. M., Sandford, S. A., \& Tielens, A. G. G. M. 2004, ApJ, 604, 252

Salama, F., Bakes, E. L. O., Allamandola, L. J., \& Tielens, A. G. G. M. 1996, ApJ, 458, 621

Salama, F., Galazutdinov, G. A., Krelowski, J., Biennier, L., Beletsky, Y., \& Song, I. 2011, ApJ, 728,154

Sarre, P. J. 2006, J. Mol. Spectrosc, 238, 1

Schmidt, M. W., Baldridge, K. K., Boatz, J. A., Elbert, S. T., Gordon, M. S., Jensen, J. H., Koseki, S., Matsunaga, N., Nguyen, K. A., Su, S. J., Windus, T. L., Dupuis, M., \& Montgomery, J. A. 1993, J. Comput. Chem., 14, 1347

Tielens A. G. G. M. 2008, ARA\&A, 46, 289 